\title{
Analysis of energy model and Performance of IEEE 802.15.4 WSNs under Different Duty Cycle
}

\author{
Sarvesh Kumar Singh ${ }^{1}$, Rajeev Paulus ${ }^{2}$, A.K. Jaiswal ${ }^{3}$, Mayur Kumar ${ }^{4}$ \\ ${ }^{I}$ (M.Tech scholar, Dept. of ECE/SHIATS, INDIA), ${ }^{2}$ (Assistant Prof., Dept. of ECE/SHIATS, INDIA), \\ ${ }^{3}$ (Prof. \& H.O.D, Dept. of ECE/SHIATS, INDIA), ${ }^{4}$ (Assistant Prof., Dept. of ECE/SHIATS, INDIA)
}

\begin{abstract}
IEEE 802.15.4 is an emerging communication protocol for low rate-wireless personal network (LRWPAN) which has gained importance in last few years, in practical and research field. Nowadays Zigbee used in many applications such as home automation, industrial, and medical system etc. The low rate WPANs provide low power consumption, low cost and small range wireless networking. Zigbee uses IEEE 802.15.4 as its communication protocol for medium access control (MAC) layer and physical (PHY) layer. Since in WSNs, nodes are powered by limited capacity of batteries and densely deployed in any geographic region to perform sensing. So that energy efficient operation and better QoS are always desired in WSNs. In this paper, we have focused on two energy model i.e. Mica mote and mica $z$ and beacon enable mode operation of IEEE802.15.4 with different duty cycle under different traffic load. The duty cycle operation achieve by setting two system parameters, macBeaconOrder (BO) and macSuperframeorder (SO). This paper, comparatively analyzed the energy model and performance of IEEE 802.15.4 with different percentage of duty cycle on the basis of energy consumption and QoS parameter. The matrices used in performance evaluation are packet loss rate (PLR), average end to end delay, throughput, residual battery capacity and energy consumption using Qualnet 6.1 simulator.
\end{abstract}

Keywords -WSN, IEEE 802.15.4, Bacon order (BO), Super frame order (SO), Duty Cycle, CBR traffic, and Qualnet 6.1.

\section{Introduction}

Wireless sensor network (WSN) is an emerging technology due to advancement made in wireless communication, silicon micro machining and information technology. Wireless sensor networks consist of large scale networks of small embedded devices, each having sensing, communication and computation capabilities [1]. In wireless sensor networks (WSNs), sensor nodes are randomly deployed in any geographical region, which performs sensing mechanism, collect information and process information to sink node [2].

Fig.1 represents the system architecture of sensor node. A sensor node consists of five main components. Controller which is used to process all relevant data, memory is used to store program and intermediate data, sensor as sensing unit, transceivers for sending and receiving information over wireless channel and battery source as the power unit. The hardware devices in sensing unit consist of several sensors, which recorded the measurable changes in the physical condition. The measurable changes are may be vibration, temperature, sound, motion, or pressure in environmental conditions. There are basically two type of sensor node used in sensor network. One is normal sensor node which is deployed to sense the physical phenomenon. The other is the gateway node that interfaces sensor network to the external world.

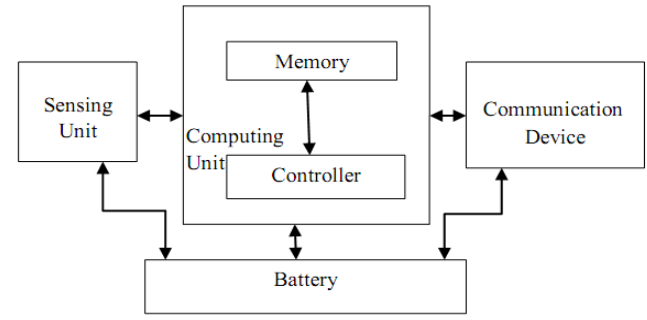

Fig.1. Sensor node system architecture [3].

Since in WSN nodes are powered by non-rechargeable battery and scavenging of node is very difficult. So that network life time is limited. Therefore it is necessary to focus on energy efficient operation such as energy model, communication protocol, modulation and routing etc.

In WSN, for energy efficient operation zigbee is an emerging technology for wireless personal area network (WPANs). Zigbee uses IEEE 802.15.4 standard as its communication protocol for medium access control layer and physical layer. IEEE 802.15.4 is a standard for LR-WPAN which provide low data rate, low power consumption and secure short range networking at low cost. The IEEE 802.15.4 MAC standard achieves duty cycle operation by setting two parameter macbeaconorder (BO) and macsuperframe order (SO). Wireless 
sensor network has many applications in every field such as in military operation, home automation, environmental application, logistic management, health application etc [4].In this paper, we consider all above parameter to evaluate network performance such as energy consumption and QoS with different percentage of duty cycle under different packet rate.

The rest of paper is organized as follows: in section II, overview of IEEE802.15.4 is given. In section III, we explain related work. In section IV, simulation set up is included. In section V, result and discussion is given. Finally, keeping all this in mind, we concluded our result in section VI.

\section{Overview of IEEE 802.15.4}

It is emerging standard for low rate wireless personal area network (LR-WPAN). It is designed to provide low data rate, low power consumption, and low cost wireless networking. It provides low cost connectivity for the sensor nodes which needs long battery life or low power consumption operation. The IEEE 802.15.4 standard mainly define the physical (PHY) and medium access control (MAC) layers characteristic for low rate wireless personal area networks (LR-WPANs). In IEEE 802.15.4 the physical layer provides an interface between the MAC layer and the physical radio channel.

\subsection{IEEE 802.15.4 Physical (PHY)}

In IEEE 802.15.4 the physical layer provides an interface between the MAC layer and the physical radio channel. The PHY layer is responsible for activation and deactivation of radio transceiver according to request obtained from MAC layer, energy detection, link quality indication, clear channel assessment and channel frequency selection as well as receiving packet across physical medium. In IEEE 802.15.4, physical layer support three frequency bands with 27 radio channel. The frequency bands are $2450 \mathrm{MHz}, 915 \mathrm{MHz}$ and 868 $\mathrm{MHz}$ with 16,10,1 channels respectively [6]. The entire band using direct sequence spread spectrum (DSSS) access mode, band $2450 \mathrm{MHz}$ uses offset quadrature phase shift keying (O-QPSK) modulation scheme and band 915/868 MHz uses BPSK and ASK modulation schemes. The physical layer specification including frequency band and data rate parameter is indicated in table 1.

Table 1.Frequency band and data rate.

\begin{tabular}{|l|l|l|l|}
\hline $\begin{array}{l}\text { Frequency } \\
\text { Band(MHz) }\end{array}$ & Modulation & $\begin{array}{l}\text { Bit Rate } \\
(\mathbf{k b} / \mathbf{s})\end{array}$ & Symbols \\
\hline $868-868.6$ & BPSK & 20 & Binary \\
\hline $868-868.6$ & ASK & 250 & 20 -bit PSSS \\
\hline $902-928$ & BPSK & 40 & Binary \\
\hline $2400-2483.5$ & O-QPSK & 250 & 16 -ary Orthogonal \\
\hline
\end{tabular}

\subsection{Network Devices And Topology}

IEEE 802.15.4 consists of two types of devices which can be FFDs (full function devices) and RFDs (reduced function devices). The FFD can operate in three modes such as a PAN coordinator, a simple coordinator or a device. An FFD can communicate to RFDs or other FFDs but RFD can communicate only to FFD. The RFDs only act as end devices which sense the event and send sensed information to coordinator or PAN coordinator. An IEEE 802.15.4 supported three type of network topology: the star topology, mesh topology and tree topology. In star topology, devices are interconnected in form of star in which central node is PAN coordinator and other RFDs, FFDs directly communicate to PAN. In mesh topology, devices communicate with other devices placed in its radio range. The structure of different topology depicted in fig. 2.

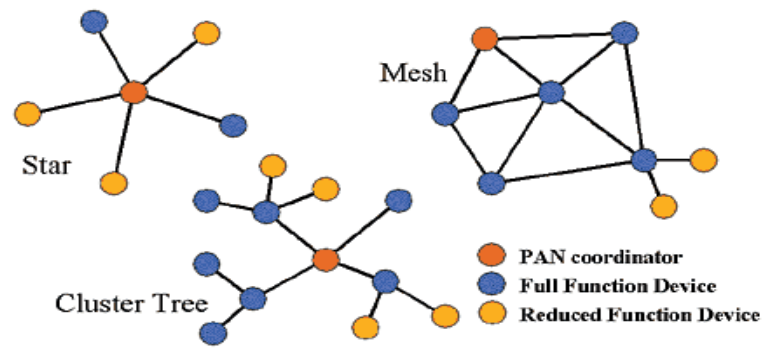

Fig. 2.Topology supported by IEEE 802.15.4

\subsection{IEEE 802.15.4 MAC}

In IEEE 802.15.4 the MAC layer is responsible for association and disassociation of device, manages super frame, control channel access, validates frame, handling and maintaining time slot mechanism. The IEEE 802.15.4 MAC layer mechanism is based on CSMA/CA protocol. IEEE 802.15.4 supports two modes of operation, beacon enabled and non beacon-enabled mode operation. For beacon enabled mode it uses slotted 
CSMA/CA and unslotted CSMA/CA is used for beaconless mode. In beacon enabled mode, a PAN coordinator periodically generates beacon frame that provide synchronization, and slots to RFDs for data transmission. In non beacon-enable mode, all RFDs send their data to sink node without synchronization, which does not provide guarantee time to deliver data. Beacon enable mode is completely defined by a structure called superframe structure. The superframe structure shown in fig. 3. Super frame consists two periods, active and inactive period. Further the active period is sub divided in to contention access period (CAP) and contention free period (CFP). The PAN coordinator transmitted beacon at slot 0, after the completion of beacon CAP will start using CSMA/CA algorithm. The FFDs which require the fixed rate of transmission can asked for guaranteed time slot (GTS) from the coordinator. A coordinator can allocate at most seven GTS to network devices. Superframe provides a duty cycle operation by setting two system parameter, macbeaconorder (BO) and macsuperframeorder(SO), such that low power operation is achieved for deployed network [5].

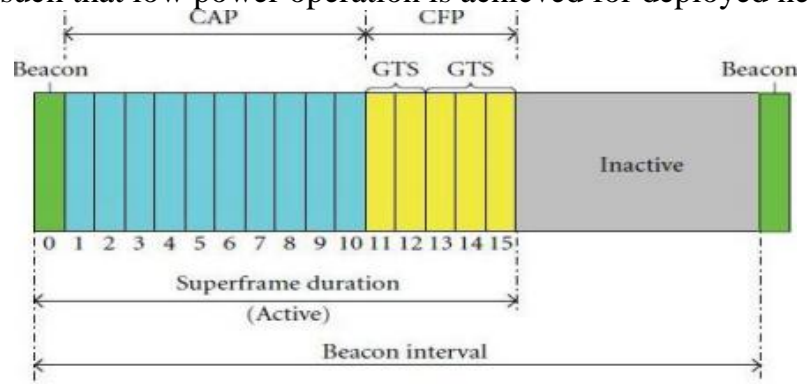

Fig. 3.Superframe structure

The superframe active period also called as superframe duration (SD). The superframe duration (SD) and beacon interval (BI) for given $\mathrm{BO}$ and $\mathrm{SO}$ are defined as follows.

$\mathrm{BI}=\mathrm{aBaseSuperFrameDuration} \times 2^{\mathrm{BO}}$

$\mathrm{SD}=$ aBaseSuperFrameDuration $\times 2^{\mathrm{SO}}$

For beacon enabled operation $\mathrm{SO}$ and $\mathrm{BO}$ should satisfy the relationship $0 \leq \mathrm{SO} \leq \mathrm{BO} \leq 14$, and for non beacon mode $\mathrm{BO}=\mathrm{SO}=15$. Low power operation for network is achieved by choosing low duty cycle. The duty cycle is calculated as.

Duty cycle $=(2)^{\text {SO-BO }} \times 100 \%$

Therefore, each device will be active for $(2){ }^{\text {SO-BO }}$ portion of time, and sleep for 1-(2) ${ }^{\text {SO-BO }}$ portion of time. A large duty cycle result in higher power consumption as devices remains in active state for longer periods.

\section{Related Work}

In the past, several researches have been done to analyze the performance of wireless sensor network using IEEE 802.15.4 (LR-WPAN) protocol.

Zukarman, et al [8] proposed C-based simulation model for beacon enable IEEE 802.15 .4 by varying the MAC macBeaconOrder (BO) and macSuperframeOrder (SO) such that the energy consumption of network is minimized.

Jelena, et al [9] modeled the operation of MAC layer for beacon enabled IEEE 802.15.4 network with both uplink and downlink traffic.

Zhang, et al [10] proposed an analytical model for the energy efficiency of the IEEE 802.15.4 slotted CSMA/CA to save power and enhanced throughput of the network.

Feng, et al [11] analyzed the throughput of slotted and unslotted CSMA are almost equal when normalized propagation delay is much less than 1 and the basic time period (BTP) is equal to the propagation delay.

Kouba, et al [12] proposed a time division beacon scheduling (TDBS) mechanism for ZigBee clustertree wireless sensor networks because Synchronization is a challenging and important issue for time-sensitive Wireless Sensor Networks (WSN). In this mechanism beacons are transmitted by coordinator on time division basis.

Mohanty, et al [13] analyzed QoS parameter in IEEE 802.15.4 mesh networking using MANET routing under different loads.

Rihan, et al [14] analyzed the effect of co-existence ISM frequency band in zigbee and IEEE802.11b/g LAN using OPNET V.16 simulator

Yuan, et al [15] analyzed mobility aware node deployment in zigbee to increase packet delivery ratio using ns-2 network simulator.

\section{Simulation Setup}

Simulation is carried out by Qualnet 6.1 network simulator with CBR traffic application. The CBR traffic application provides constant bit rate throughout the process. In the scenario, we have randomly deployed ten 
sensor nodes in single hop star topology manner. The central node is a sink node which is acting as a PAN coordinator. The sink node is FFD, which collect the sensed information from various sensor nodes. Meanwhile, the remaining nodes are worked as sensor node or end devices. The end devices are RFDs, which sensed the measurable changes in environment and send information to sink node. There are nine CBR applications are laid down between sink and source. All sensor nodes connected to wireless subnet to propagate sensed information across the network. All nodes are deployed in the network in such a way that there is no hidden node in the network. The parameters which are considered in the simulation are energy model and superframe duty cycle by varying macbeaconorder (BO) apart from these packet rates is also varied. To route the packet from source to destination we choose AODV as routing protocol [7]. The configured simulation parameters are given in table 2.With the help of simulation result, we have analyzed throughput, packet loss rate, average end to end delay, residual battery capacity and energy consumption in four modes i.e. in transmit, receive, Idle and sleep mode. These performance metrics are defined below:

\subsection{Throughput}

It is defined as average rate of successful packet received at its destination. It is measured in bits per second (bits/second). It is calculated under application layer at CBR server.

\subsection{Packet Loss Rate}

It is the fraction of number of packet dropped to the total number of packet generated by all devices. It is the measure of reliability of protocol and network.

\subsection{Average End To End Delay}

It is defines as time taken by a packet to travel across the network from source to destination. It is caused due to route discovery, queuing and propagation time. It is measured in second.

\subsection{Residual Battery Capacity}

It is indicated the remaining capacity of battery after running the scenario.

\subsection{Energy consumption}

It is defined as the amount of energy consumed during different modes of sensor nodes i.e. in transmit mode, receive mode, idle mode and sleep mode.

Table-2 Configured parameter.

\begin{tabular}{|l|l|}
\hline Simulation Parameters & Corresponding Values \\
\hline Channel frequency & $2.4 \mathrm{GHz}$ \\
\hline Simulation time & $500 \mathrm{sec}$ \\
\hline Radio Type & IEEE 802.15 .4 radio \\
\hline Packet reception model & PHY 802.15 .4 \\
\hline MAC protocol & IEEE 802.15 .4 \\
\hline CCA mode & Carrier-sense \\
\hline Antenna type & Omni directional \\
\hline Routing Protocol & AODV \\
\hline Network Protocol & IPv4 \\
\hline Beacon order & $1,2,3$ \\
\hline Super frame order & 1 (fixed) \\
\hline Application & CBR \\
\hline $\begin{array}{l}\text { Packet rate (packet per } \\
\text { second) }\end{array}$ & $1,2,4,10,20$ \\
\hline Energy Model & MICA MOTE/MICA Z \\
\hline Battery model & Duracell AA \\
\hline
\end{tabular}

\section{Result And Discussion}

Fig.4 shows that variation of throughput against different percentage of duty cycle. It shows that at lower duty cycle throughput is less and at higher duty cycle more throughput is obtained for same packet rate. When the packet generation rate is increases means larger number of packet send per second, higher throughput is obtained in all duty cycle, since more bits per second are transferred. 


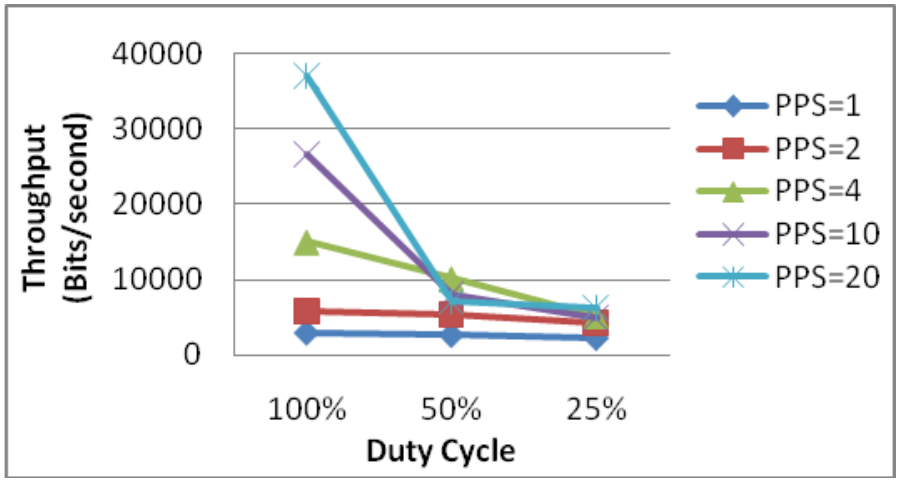

Fig. 4. Duty Cycle vs Throughput

Fig 5.Shows the packet loss rate in different percentage of duty cycle. It shows that at higher duty cycle the packet loss rate is decreases and at lower duty cycle probability of packet loss is increases. When packet generation rate is increases means traffic on network is increases then at higher duty cycle collision probability is less due to availability of high bandwidth. At high traffic load the collision probability is more in low duty cycle, hence packet loss ratio increases.

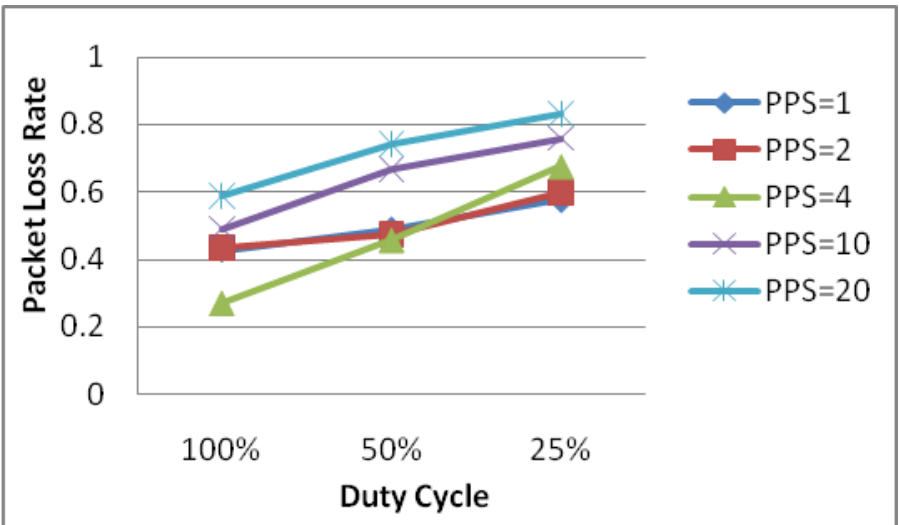

Fig. 5. Duty Cycle Vs PLR

Fig 6. Show that average end to end delay against different percentage of duty cycle. It shows that higher delays are experienced for lower duty cycle. The reason is that lower duty cycle causes longer inactive period, in which buffered packet experienced longer sleeping delay. The higher packet rate causes the heavy traffic load on the network due to which more delay will be seen, and may cause more collision and retransmissions of the packets.

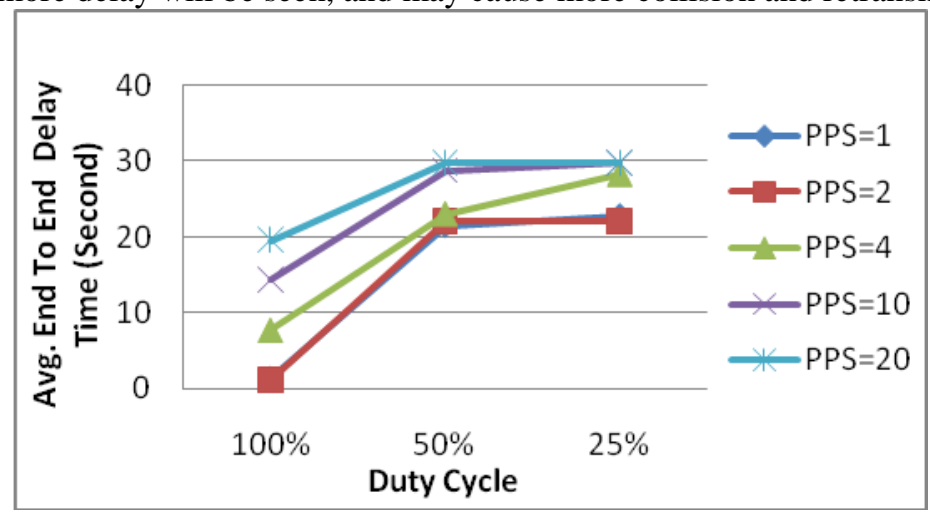

Fig. 6. Duty Cycle vs Average end to end Delay

Fig. 7 shows that battery capacity in different duty cycle. It shows that at higher duty cycle more battery power is consumed, this is caused due to longer wake period of nodes. Meanwhile, at lower duty cycle less energy is consumed due to lower wakeup period of nodes. 


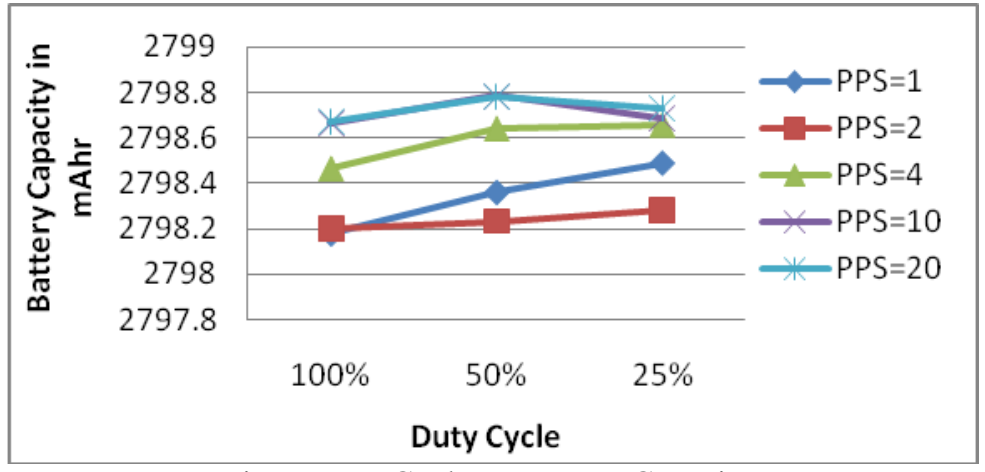

Fig. 7.Duty Cycle vs Battery Capacity.

Fig. 8 shows that in transmit mode, mica mote consume less energy than mica z mote in all percentage of duty cycle. So in transmit mode, mica mote energy model is more efficient than mica $z$ energy model.

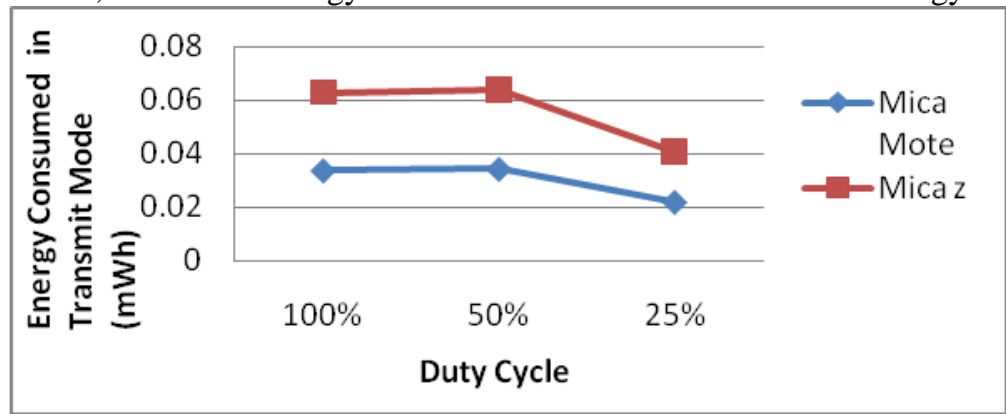

Fig. 8. Energy Consumption In Transmit Mode.

Fig. 9 shows that in receive mode, energy consumption in mica mote is linearly decreases as duty cycle decreases, but energy consumption in mica $\mathrm{z}$ is more at $100 \%$ duty cycle and least at lower duty cycle compared to mica mote.

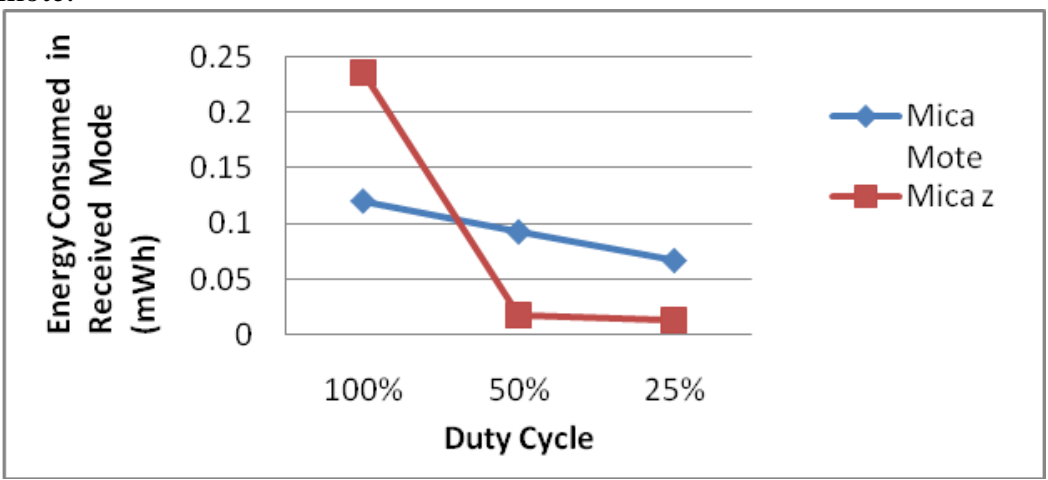

Fig. 9. Energy Consumption In Receive Mode

Fig. 10 shows that in idle, mica mote consume more energy than mica $z$. so that in idle mode, mica $\mathrm{z}$ energy model is more energy efficient then mica mote energy model.

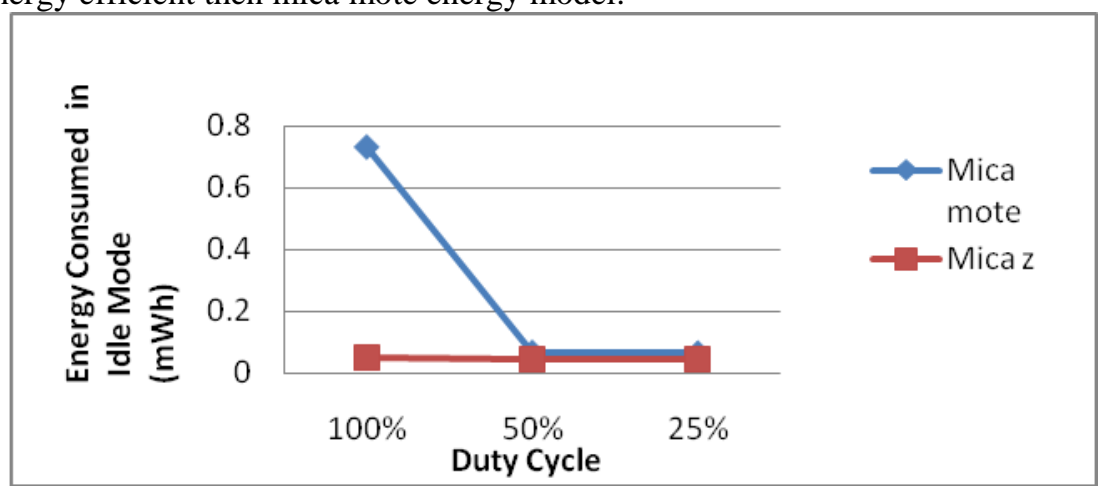

Fig. 10. Energy Consumption in Idle Mode

Fig. 11 shows that in sleep mode, mica $\mathrm{z}$ is consuming very less energy and it is more efficient than mica mote energy model. 


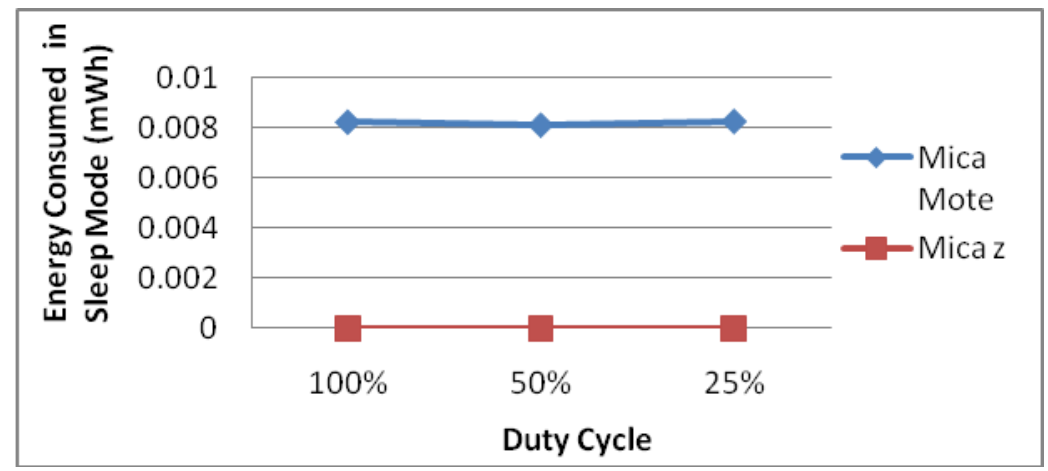

Fig. 11. Energy Consumption In Sleep Mode

\section{Conclusion}

In this cross layer approach (PHY/MAC), we have analyzed WSNs and energy model under different percentage of duty cycle with IEEE 802.15.4 standard. We have calculated different energy consumption and QoS parameter. We found that under different traffic loads QoS parameter performance is better with higher duty cycle and it degraded with lower duty cycle. But in the perspective of energy consumption lower duty cycle performance is better as compared to higher duty cycle and it can enhanced lifetime of the network. So that there is a trade of exist between QoS and energy consumption. This paper is also focused on energy model i.e. Mica mote and Mica z. Since in WSNs nodes are operated in four operational mode i.e. transmit, receive, idle and sleep mode. So collectively, by analyzing the result we have concluded that in these four operational modes mica z energy model performance is better than mica mote energy model in term of total power consumption.

\section{Reference}

[1] G. Pottie and W. Kaiser, "Wireless integrated network sensors," ACM Communications, vol. 43, no. 5, pp. 51-58, May 2000

[2] I. F. Akyildiz, W. Su, Y. Sankasubramaniam, and E. Cayirci, "Wireless Sensor Networks: A Survey", Computer Networks, 38: 393-422, 2002.

[3] J. Shaw, E. Silverberg, M. Al-Kateb, "Simulation of Large-Scale Wireless Sensor Networks", pg57, 2008.

[4] K. Sohraby, D. Minoli and T. Znati, "Wireless Sensor Network Technology, Protocols and Applications", John Wiley \& Sons, $\operatorname{pg} 10-11,2007$

[5] Y.-Kai Huang; Ai-Chun Pang; Hui-Nien Hung; "A comprehensive analysis of low-power operation for beacon-enabled IEEE 802.15.4 wireless networks" IEEE Transactions on Volume: 8, Issue: 11 November 2008.

[6] Qualnet User's Guide.

[7] Perkins, C., Belding-Royer, E. and Das, S.; “Ad hoc on-Demand Distance Vector (AODV) Routing”, RFC 3561 (rfc3561) Pg. 1-37, July2003

[8] F. Shu, T. Sakurai, H. L. Vu, and M. Zukerman, "Optimizing the IEEE 802.15.4 MAC," in Proc. IEEE Region 10 Conference (TENCON), Nov. 2006

[9] J. Misic, S. Shaf, and V. B. Misic, "Performance of a beacon enabled IEEE 802.15.4 cluster with downlink and uplink traffic," IEEE Trans. Parallel and Distributed Syst., vol. 17, pp. 361-376, Apr. 2006

[10] Y. Zhang, P.Xu, G. Bi and F.S Bao, “ Analysis of energy efficiency and power saving in IEEE 802.15.4”, IEEE Wireless Communications and Networking Conference, pp. 3330-3334, 2007.

[11] W. Feng, L. Dou, and Z. Yuping, “Analysis and compare of slotted and unslotted CSMA in IEEE 802.15.4," in Proceedings of the 5th International Conference on Wireless Communications, Networking and Mobile Computing (WiCOM '09), pp. 3659-3663, September 2009.

[12] A. Kouba , A. Cunha, M. Alves, and E. Tovar, “TDBS: A Time Division Beacon Scheduling Mechanism for Zigbee Cluster-Tree Wireless Sensor Networks," Real-Time Systems J., vol. 40, no. 3, pp. 321-354, Dec. 2008

[13] S. Mohanty, and .S.K.Patra, "Quality of Service Analysis in IEEE 802.15.4 Mesh Networks using MANET", IEEE conference. On ICCCNT pp.1-7, 2010

[14] M. Rihan, M. El-Khamy and M. El-sharkawy, "on zigbee co-exitance in ISM band; measurement and simulation," IEEE international conf. on ICWCUCA, pp.1-6, 2012.

[15] Y. yuan, W.H. chung, P.cheng and A. pang, "A mobality node deployement and tree construction frame work for zigbee wireless network", IEEE transaction on vehicular technology, vol. 62, issue 6, pp.2763-2779, 2013. 